\title{
Analysis of Proteus mirabilis Distribution in Multi-Species Biofilms on Urinary Catheters and Determination of Bacteria Resistance to Antimicrobial Agents
}

\author{
MAGDALENA MORYL ${ }^{1 *}$, AGNIESZKA TORZEWSKA로 PAWEł, JAŁMUŻNA² and ANTONI RÓŻALSKI \\ ${ }^{1}$ Department of Immunobiology of Bacteria, Institute of Microbiology, Biotechnology and Immunology \\ University of Łódź, Łódź, Poland \\ ${ }^{2}$ Department of Urology and Transplantology of Kidneys, M. Pirogow Specialist Hospital, Eódź, Poland
}

Submitted 11 March 2013, accepted 16 November 2013

\begin{abstract}
The objectives of the investigation presented in this paper were: to examine the frequency of P. mirabilis isolation from catheters and assess the complexity of multi-species biofilms which these bacteria form, as well as to determine the vulnerability of planktonic and sessile P. mirabilis populations to popular antibiotics and compare it to the susceptibility of other Gram-negative bacteria isolated as associated flora from multi-species biofilm. 88 urological catheters, collected from long-term catheterized patients were examined. Uropathogens were recovered from the catheter surface by sonication, and identified on standard diagnostic media. The broth-microdilution method and the MBEC High-throughput Screening assay were used to determine the bacterial resistance to antibiotics. 279 microorganisms were isolated from 88 urinary catheter biofilms. The Enterobacteriaceae family were the most frequently detected bacteria (53.2\% of isolates), whereas Proteus spp. isolation accounted for $17.9 \%$, which placed these bacilli on the third position in the Enterobacteraceae family. Among all the tested drugs, amikacin and cephalosporins (ceftriaxone, cefotaxime and cefaclor) exhibited the highest activity against $P$. mirabilis planktonic cells, $86 \%$ and $73 \%$ of strains were susceptible to these antibiotics, respectively. $100 \%$ of $P$. mirabilis sessile forms were resistant to cefepime, ciprofloxacin, gatifloxacin, and norfloxacin. Amikacin and ceftriaxone affected only $5 \%$ of sessile forms. The planktonic cells of the other studied uropathogens were mostly vulnerable to the all tested drugs (exception P. aeruginosa strains), the most effective of which occurred to be amikacin and cefepime. Obtained MBECs values were 2-512-fold higher than MICs assessed for planktonic forms.
\end{abstract}

Ke y word s: Proteus mirabilis, antibiotic resistance, associated flora, biofilm, urinary catheter

\section{Introduction}

Complicated urinary tract infections (UTIs) are associated with structural or functional abnormalities in the urinary tract such as a catheterization, calculi, immunosupression and others (Jacobsen, 2008). Probability of developing the infection is similar in women and men and in all age groups (Pallet and Hand, 2010). Devices, e.g. indwelling catheters, which are inserted in the urinary tract facilitate microbial colonization. Bacteria attach to outer and inner surfaces of a catheter and form biofilm, which is responsible for $65-85 \%$ cather associated UTIs. $\Lambda$ fter a few days of catheterization multi-species biofilm, consisting of several organisms, is often isolated (Muzzi-Bjornson and Macera, 2011). Species like: Enterococcus faecalis, Staphylococcus epidermidis, Escherichia coli, Proteus mirabilis, Pseudomonas aeruginosa, Klebsiella pneumoniae are most frequently detected on the catheter surface (Niveditha etal., 2012). The structure of multi-species biofilm is different from biofilm formed by one species of bacteria. The interactions between microorganisms which form this structure change biofilm physiology and function, facilitating the horizontal gene transfer. Consequently, in multi - species biofilms resistance to antimicrobial agents and host defense is observed more frequently (Yang et al., 2011). Biofilm age, its density and the extent of the surface which it covers are the main factors affecting its susceptibility to antimicrobials. It is possible that microorganisms in biofilm use a number of mechanisms responsible for their resistance. The first one is slow or incomplete penetration of antimicrobial agents into biofilm. A significant role in this process is played by a sticky matrix which covers bacterial cells and prevents the diffusion of drugs to the target by binding them or influencing the rate of

\footnotetext{
* Corresponding author: M. Moryl, Department of Immunobiology of Bacteria, University of Lódź, Banacha 12/16, 90-237 Lódź,
} Poland; phone: (00-48-42) 6354324; fax: (00-48-42) 6355818; e-mail: magdabar@biol.uni.lodz.pl 
molecules transport to the biofilm interior. An explanation for high biofilm resistance could be the reduction of the metabolic activity of sessile bacterial cells. A significant role in biofilm resistance to antibiotics is played by persisters: a spore-like cells, dormant and incapable of growth (Hoiby et al., 2010, Lazar and Chifiriuc, 2010). The main mechanism is still undetermined, recognition of this process will help to develop new methods of preventing biofilm formation and matriculation processes. Numerous mechanisms of biofilm resistance cause that concentrations of antibacterial agents needed for biofilm eradication are 1000-1500 times higher than concentrations needed to eradicate planktonic bacteria of the same species (Chen and Wen, 2011). Urea-splitting bacteria: Proteus spp., Providencia stuartii and Morganella morganii are often detected on indwelling catheter surfaces. The presence of these microorganisms leads to many complications such as formation of biofilm encrusted with calcium and magnesium phosphates crystals, which results in catheter blockage (Pallett and Hand, 2010). High ability of urease synthesis is the main reason why Proteus infections cause more cytological damage in renal tissue, than those developed by E. coli (Wilde et al., 2013). This dangerous pathogen, despite the fact that it is often isolated from urological catheters, still remains inadequately characterized. Therefore, the first objective of the investigations presented in this paper was to examine the frequency of P. mirabilis isolation from catheters and assess the complexity of multi-species biofilm formed by these bacteria. The next aim was to determine the susceptibility of P. mirabilis (planktonic and biofilm populations) to antibiotics. Finally, the vulnerability of sessile and planktonic cells of other Gram-negative bacteria (K. pneumoniae, E. coli, Enterobacter cloacae, P. stuartii, M. morganii, P. aeruginosa) was compared to that of P. mirabilis.

\section{Experimental}

\section{Materials and Methods}

88 urological catheters, obtained from long-term catheterized patients - mostly men aged 41-91 years and three women, were examined in this study. Patients had been catheterized for the periods of a few months to a few years, however, each catheter was replaced every two weeks. Most frequent indication for catheterization in older patients was benign prostatic hypertrophy, in younger ones: stroke, multiple sclerosis or spine damage. Patients were treated in two outpatient clinics in Łódź. In the case of urinary tract infection (30\% of patients), the patients were subjected to treatment with antibiotics: ciprofloxacin, norfloxacin, nitrofurantoin, sometimes antifungal therapy (nystatin, fluconazole) was implemented. Information about patients from whom studied strains were collected is presented in Table I.

Strains used in this research were stored at $-80^{\circ} \mathrm{C}$ in Tryptone Soya Broth (TSB) and in dimethyl sulfoxide (DMSO), and cultivated on TSA plates for $24 \mathrm{~h}$ at $37^{\circ} \mathrm{C}$. E. coli ATCC 25922 was used as the reference strain.

Bacteria from the catheter surface were recovered by sonication of the catheter fragments. Obtained sus-

Table I

Characteristics of patients from whom bacterial strains were isolated.

\begin{tabular}{|c|c|c|c|}
\hline Bacteria & $\begin{array}{c}\text { Strains } \\
\text { no. }\end{array}$ & $\begin{array}{c}\text { Gender/ } \\
\text { patient } \\
\text { age }\end{array}$ & Disease \\
\hline \multirow[t]{22}{*}{ Proteus mirabilis } & 5 & $\mathrm{M} / 72$ & $\mathrm{BPH}$ \\
\hline & 6 & M/92 & BPH \\
\hline & 7 & $\mathrm{M} / 77$ & $\mathrm{BPH}$ \\
\hline & 8 & $\mathrm{M} / 83$ & diabetes \\
\hline & 9 & $\mathrm{M} / 77$ & $\mathrm{BPH}$ \\
\hline & 11 & $\mathrm{M} / 89$ & $\mathrm{BPH}$ \\
\hline & 12 & $\mathrm{M} / 73$ & $\mathrm{BPH}$ \\
\hline & 15 & $\mathrm{M} / 82$ & $\mathrm{BPH}$ \\
\hline & 20 & $\mathrm{M} / 86$ & stroke \\
\hline & 24 & $\mathrm{M} / 41$ & multiple sclerosis \\
\hline & 31 & $\mathrm{M} / 57$ & spine damage \\
\hline & 32 & $\mathrm{M} / 80$ & stroke \\
\hline & 33 & $\mathrm{M} / 88$ & $\mathrm{BPH}$, stroke \\
\hline & 34 & W/73 & stroke, diabetes \\
\hline & 41 & $\mathrm{M} / 71$ & $\mathrm{BPH}$ \\
\hline & 44 & $\mathrm{M} / 81$ & stroke \\
\hline & 46 & $\mathrm{M} / 81$ & $\mathrm{BPH}$, stroke \\
\hline & 57 & $\mathrm{M} / 71$ & renal failure \\
\hline & 70 & $\mathrm{M} / 80$ & $\mathrm{BPH}$ \\
\hline & 71 & $\mathrm{M} / 81$ & stroke \\
\hline & 77 & $\mathrm{M} / 67$ & $\mathrm{BPH}$ \\
\hline & 84 & $\mathrm{M} / 71$ & urethral structure \\
\hline \multirow[t]{2}{*}{ Morganella morganii } & 42 & $\mathrm{M} / 72$ & $\mathrm{BPH}$ \\
\hline & 67 & $\mathrm{M} / 79$ & $\mathrm{BPH}$, stroke \\
\hline \multirow[t]{2}{*}{ Enterobacter cloacae } & 31 & $\mathrm{M} / 57$ & spine damage \\
\hline & 36 & $\mathrm{~W} / 72$ & stroke \\
\hline \multirow[t]{2}{*}{ Providencia stuartii } & 9 & $\mathrm{M} / 73$ & $\mathrm{BPH}$ \\
\hline & 71 & $\mathrm{M} / 81$ & stroke \\
\hline \multirow[t]{2}{*}{ Klebsiella pneumoniae } & 46 & $\mathrm{M} / 81$ & $\mathrm{BPH}$, stroke \\
\hline & 56 & $\mathrm{M} / 80$ & retention of urine \\
\hline \multirow[t]{2}{*}{ Pseudomonas aeruginosa } & 11 & $\mathrm{M} / 89$ & $\mathrm{BPH}$ \\
\hline & 46 & $\mathrm{M} / 81$ & $\mathrm{BPH}$, stroke \\
\hline \multirow[t]{2}{*}{ Escherichia coli } & 9 & $\mathrm{M} / 73$ & $\mathrm{BPH}$ \\
\hline & 71 & $\mathrm{M} / 81$ & stroke \\
\hline
\end{tabular}

$\mathrm{BPH}$ - benign prostatic hypertrophy; M - man; W - woman 
pensions were plated on blood and McConkey agars. Next, the identification of morphologically different colonies was performed. The microscopic slide and culturing the bacteria in different identification media were carried out using traditional diagnostic media and API $20 \mathrm{E}$ and NE tests. Antimicrobial sensitivity of P. mirabilis planktonic cells was examined by the standard broth-microdilution method by Clinical and Laboratory Standards Institute (CLSI). The MICs (minimum inhibitory concentrations) and MBCs (minimum bactericidal concentrations) were determined in Mueller Hinton broth, by reading the turbidity in wells. Antimicrobials: amoxicillin and clavulanic acid AMC (Polfa), cefazolin CFZ, cephalexin LEX, cefuroxime CXM, cefotaxime CTX, cefaclor CEC, ceftriaxone CRO, gentamicin GM, amikacin AMK (Sigma), ciprofloxacin CIP (Fluca), ofloxacin OFX, norfloxacin NOR (Sigma) and cotrimoxazole SXT (Polfa) were used in the test.

Biofilms susceptibility to antimicrobial agents was studied using the MBEC (minimum biofilm eradication concentration) High-throughput Screening assay (Calgary Alberta, Canada). Antibiotics with the highest activity toward P. mirabilis planktonic cells (in the broth-microdilution method) were chosen: amikacin, cephalexin, ceftriaxone; and ciprofloxacin - fluoroquinolone often used in Poland to treat urinary tract infections and the drugs which are not used in the Polish medical practice: cefepime FEP and gatifloxacin GAT (Bristol Squibb Myers).

The test was performed according to the previously described method by Ceri et al. (1999), which consists of three stages: 1. Biofilm formation; 2. MIC values determination, 3. MBEC values determination.

\section{Results}

279 microorganisms were isolated from 88 urinary catheter biofilms and classified into 28 species. Over $90 \%$ of the isolates were classified into three groups: Enterobacteriaceae family - the most frequently isolated bacteria - 53.2\%, Pseudomonadaceae family - $21.7 \%$ and Streptococcus group D - 21.3\% (Fig. 1A). Distribution of different genera from the Enterobacteriaceae family is summarized in Fig. 1B. E. coli was the most often isolated species, whereas Proteus spp., (17.9\% of isolates) was ranked in the third position. Among Proteus isolates, $P$. mirabilis species was detected most frequently ( $88 \%$ - 22 strains), mostly from multi-species biofilms (21 cases), composed of 2-7 microorganisms (Table II). Most common P. mirabilis associated flora involved: Streptococcus group D - in 63\% of biofilms and Pseudomonas spp. - in 59\% of biofilms. P. stuartii was often isolated together with $P$. mirabilis strains - almost 50\% of P. stuartii isolates were acquired from biofilms mixed with Proteus.

Thirteen antibiotics were tested against 22 P. mirabilis strains. The bacteria were considered sensitive or resistant on the basis of CLSI breakpoints. The reference strain - E. coli ATCC 25922 was sensitive to all the tested drugs. The resistance of $P$. mirabilis strains to antibiotics is summarized in Fig. 2.

Amikacin had the highest inhibitory and bactericidal activity against $P$. mirabilis strains. 19 out of 22 strains (86\%) were susceptible to this drug. High effectiveness of cephalosporins was also detected $-73 \%$ (16 strains) of P. mirabilis were vulnerable to ceftriaxone, cefotaxime and cefaclor. Fluoroquinolones are
A
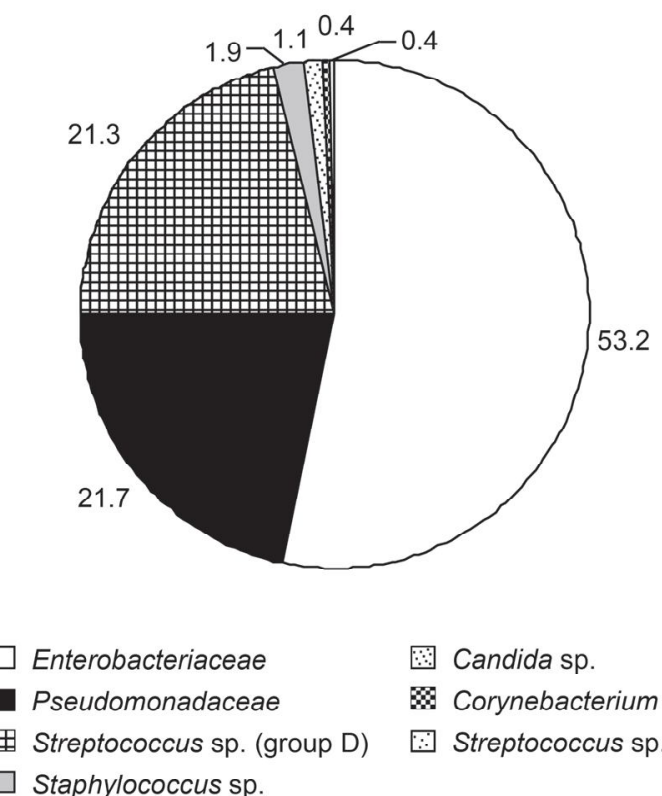

Candida sp.

\& Corynebacterium sp.

Streptococcus sp. (group B)
B

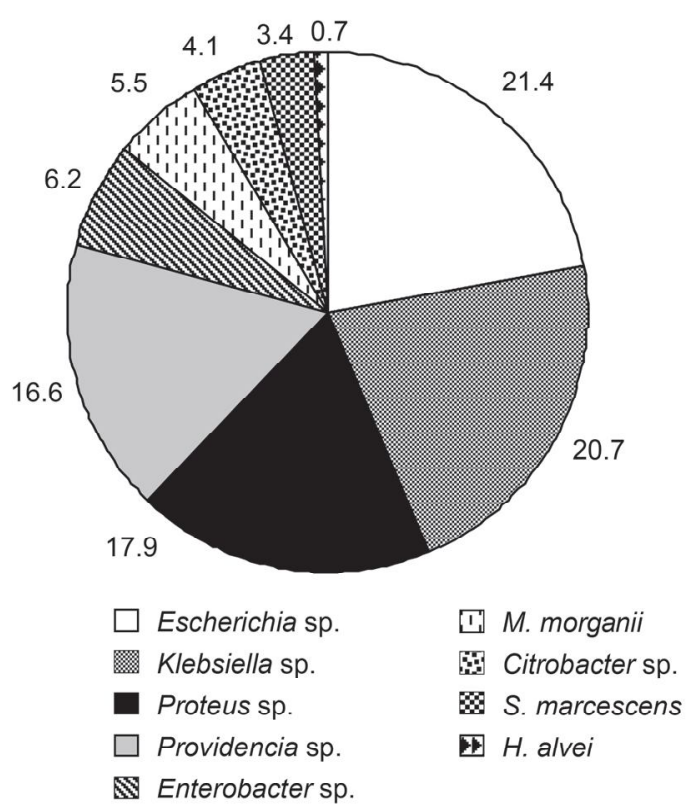

Fig. 1. Percentage of microorganisms isolated from urinary catheter biofilms (A - main groups of microorganisms, B - Enterobacteriaceae family). 
Table II

Composition of multi-species biofilms, from which Proteus mirabilis strains were isolated.

\begin{tabular}{|c|c|c|}
\hline No. & $\begin{array}{l}\text { Catheter } \\
\text { no. }\end{array}$ & Proteus mirabilis associated flora \\
\hline 1 & 5 & Pseudomonas aeruginosa, Klebsiella pneumoniae, Klebsiella oxytoca \\
\hline 2 & 6 & Pseudomonas aeruginosa, Enterococcus spp. \\
\hline 3 & 7 & Providencia stuartii, Pseudomonas aeruginosa, Klebsiella pneumoniae, Enterococcus spp. \\
\hline 4 & 8 & Citrobacter diversus, Escherichia coli, Morganella morganii, Enterococcus spp. \\
\hline 5 & 9 & Escherichia coli, Pseudomonas aeruginosa \\
\hline 6 & 11 & Escherichia coli, Pseudomonas aeruginosa, Providencia stuartii, Enterococcus faecalis \\
\hline 7 & 12 & Providencia stuartii, Pseudomonas aeruginosa, Enterococcus spp. \\
\hline 8 & 15 & Providencia stuartii, Streptococcus (group D) \\
\hline 9 & 20 & Klebsiella pneumoniae, Pseudomonas spp. Enterococcus spp. \\
\hline 10 & 24 & Providencia stuartii, Pseudomonas aeruginosa \\
\hline 11 & 31 & Providencia stuartii, Enterococcus spp. Enterobacter cloacae \\
\hline 12 & 32 & Streptococcus (group B) \\
\hline 13 & 33 & Klebsiella oxytoca, Morganella morganii, Providencia stuartii, Pseudomonas aeruginosa \\
\hline 14 & 34 & Pseudomonas aeruginosa \\
\hline 15 & 41 & Klebsiella pneumoniae, Morganella morganii, Pseudomonas aeruginosa, Streptococcus (group D) \\
\hline 16 & 44 & Escherichia coli, Providencia stuartii, Enterococcus spp. \\
\hline 17 & 46 & Pseudomonas aeruginosa, Klebsiella pneumoniae, Enterococcus spp. \\
\hline 18 & 57 & - \\
\hline 19 & 70 & Serratia marcescens, Enterococcus spp., non-fermentative OX- rods \\
\hline 20 & 71 & $\begin{array}{l}\text { Providencia stuartii, Klebsiella pneumoniae, Escherichia coli, Pseudomonas sp., Enterococcus spp. non-fermentative } \\
\text { OX- rods }\end{array}$ \\
\hline 21 & 77 & Escherichia coli, non-fermentative OX+ rods \\
\hline 22 & 84 & Escherichia coli, Klebsiella pneumoniae, Enterococcus spp. \\
\hline
\end{tabular}

very common antibiotics, which are used to treat UTIs, and ciprofloxacin is administered most frequently. In our study only 8 P. mirabilis strains were sensitive to ciprofloxacin and 5 strains were intermediate. Within the fluoroquinolones group - norfloxacin had the best efficiency ( $70 \%$ strains were vulnerable). Cotrimoxazole is usually recommended to treat bacteriuria, but most of the investigated strains were resistant (86\%) to this drug.

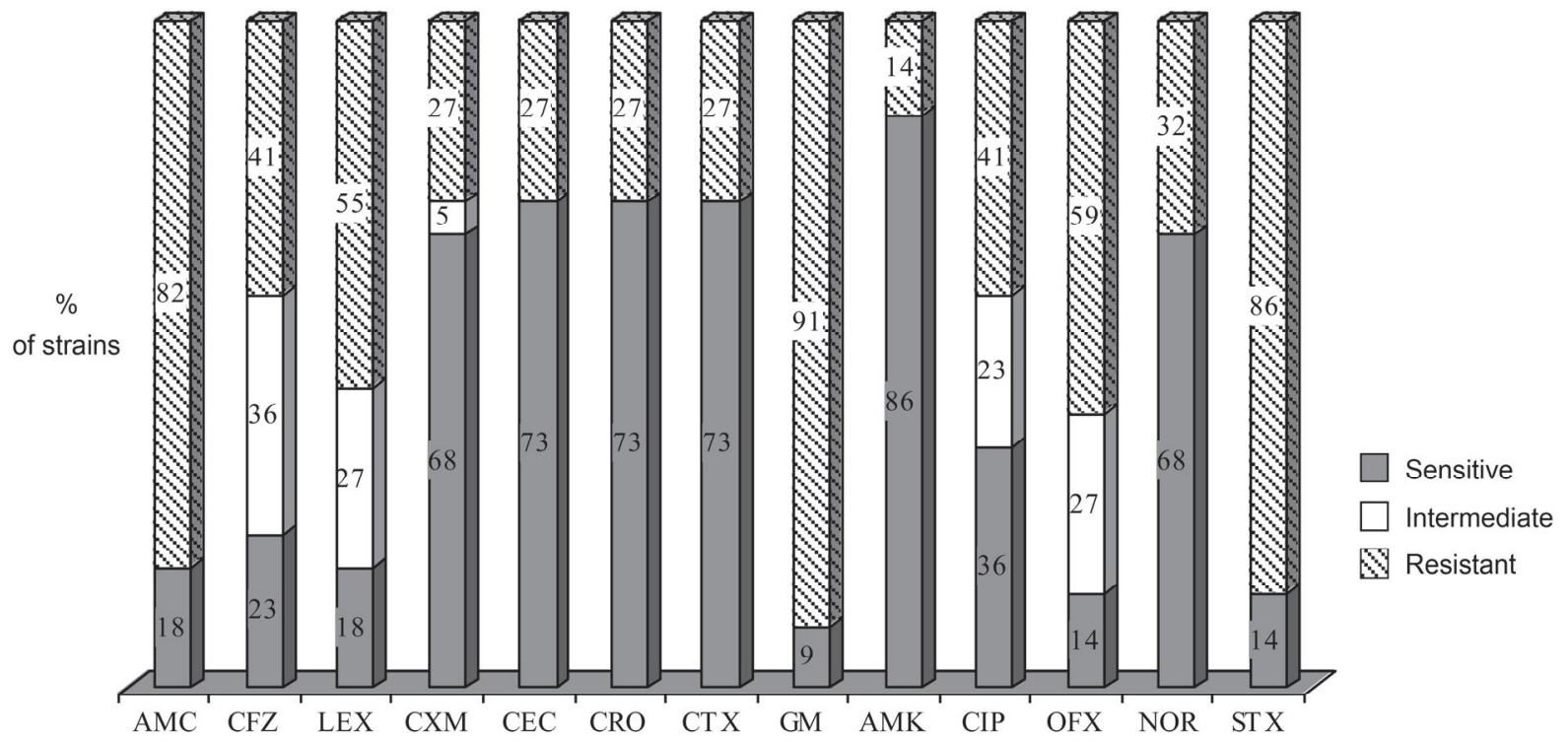

Fig. 2. Proteus mirabilis planktonic strains sensitivity to antibiotics: amoxicillin and clavulanic acid (AMC) cefazolin (CFZ), cephalexin (LEX), cefuroxime (CXM), cefotaxime (CTX), cefaclor (CEC), ceftriaxone (CRO), gentamicin (GM), amikacin (AMK), ciprofloxacin (CIP), ofloxacin (OFX), norfloxacin (NOR) and cotrimoxazole (SXT). 
Table III

Resistance of P. mirabilis planktonic and sessile populations to antibiotics.

\begin{tabular}{|c|c|c|c|c|c|c|c|c|c|c|c|c|c|c|}
\hline \multirow{2}{*}{$\begin{array}{l}\text { P. mirabilis } \\
\text { strains }\end{array}$} & \multirow{2}{*}{$\begin{array}{c}\text { Inoculum } \\
\times 10^{7} \mathrm{CFU} / \mathrm{ml}\end{array}$} & \multirow{2}{*}{$\begin{array}{c}\text { Biofilm } \\
\times 10^{6} \mathrm{CFU} / \mathrm{ml}\end{array}$} & \multicolumn{6}{|c|}{$\begin{array}{l}\text { Resistance of planktonic } \\
\text { population (MIC) }\end{array}$} & \multicolumn{6}{|c|}{ Biofilm resistance (MBEC) } \\
\hline & & & NOR & CIP & AMK & $\mathrm{CRO}$ & GAT & FEP & NOR & CIP & AMK & $\mathrm{CRO}$ & GAT & FEP \\
\hline 5 & 3.7 & 1.77 & 32 & 8 & 8 & $<1$ & 8 & $<1$ & $>1024$ & $>1024$ & 1024 & 32 & 1024 & 512 \\
\hline 6 & 7.5 & 0.62 & 2 & $<1$ & 4 & $<1$ & 16 & $<1$ & 1024 & $>1024$ & 256 & 64 & 1024 & 512 \\
\hline 7 & 5.3 & 0.23 & 4 & $<1$ & 4 & $<1$ & 2 & $<1$ & 1024 & 1024 & 1024 & 32 & 64 & 512 \\
\hline 8 & 4.8 & 2.68 & 32 & 8 & 16 & $<1$ & 8 & $<1$ & $>1024$ & $>1024$ & 1024 & 32 & 1024 & 512 \\
\hline 9 & 11.7 & 0.59 & 8 & 8 & 16 & $<1$ & 4 & $<1$ & 1024 & 1024 & 1024 & 32 & 256 & 1024 \\
\hline 11 & 4.5 & 3.05 & 16 & 8 & 8 & $<1$ & 4 & 2 & 1024 & 1024 & 128 & 64 & 1024 & 1024 \\
\hline 12 & 6.5 & 3.30 & 64 & 8 & 8 & $<1$ & 16 & $<1$ & 1024 & 1024 & 64 & 32 & $>1024$ & 1024 \\
\hline 15 & 6.1 & 3.33 & 64 & 8 & 16 & $<1$ & 4 & $<1$ & 1024 & 1024 & 32 & 32 & 1024 & 512 \\
\hline 20 & 8.45 & 1.00 & $<1$ & $<1$ & 32 & $<1$ & $<1$ & 8 & 512 & 512 & 1024 & 32 & 256 & 512 \\
\hline 24 & 6.45 & 2.83 & 16 & 8 & 8 & $<1$ & 8 & $<1$ & 1024 & 1024 & 64 & 32 & 1024 & 512 \\
\hline 31 & 14.0 & 2.01 & 16 & 8 & 16 & $<1$ & 16 & 2 & 1024 & 1024 & 64 & 8 & 1024 & 256 \\
\hline 32 & 6.1 & 1.57 & 8 & 4 & 8 & $>1024$ & 16 & 1024 & 1024 & 1024 & 16 & $>1024$ & 1024 & $>1024$ \\
\hline 33 & 10.3 & 3.91 & 128 & 128 & 16 & 8 & 64 & 16 & $>1024$ & $>1024$ & 1024 & 32 & $>1024$ & 512 \\
\hline 34 & 6.8 & 1.79 & 16 & 4 & 1024 & $>1024$ & 8 & 512 & $>1024$ & 1024 & $>1024$ & $>1024$ & 1024 & $>1024$ \\
\hline 41 & 8.2 & 1.20 & 512 & 512 & 16 & $>1024$ & 16 & 128 & $>1024$ & 1024 & 1024 & $>1024$ & 64 & $>1024$ \\
\hline 44 & 7.9 & 6.40 & 64 & 64 & 64 & 8 & 32 & 64 & $>1024$ & 1024 & 1024 & 32 & 512 & 512 \\
\hline 46 & 6.95 & 9.35 & 64 & 32 & 16 & $>1024$ & $>1024$ & $>1024$ & $>1024$ & $>1024$ & 512 & $>1024$ & $>1024$ & $>1024$ \\
\hline 57 & 2.9 & 0.97 & 32 & 8 & 16 & $<1$ & 16 & 512 & $>1024$ & 1024 & 64 & 1024 & $>1024$ & $>1024$ \\
\hline 70 & 6.0 & 9.91 & 128 & 128 & 1024 & $>1024$ & 16 & 256 & 1024 & 1024 & $>1024$ & $>1024$ & 1024 & $>1024$ \\
\hline 71 & 3.6 & 9.65 & 128 & 128 & 64 & $<1$ & 256 & 64 & $>1024$ & $>1024$ & 1024 & 32 & 1024 & 1024 \\
\hline 77 & 9.7 & 3.63 & 16 & 4 & 8 & $<1$ & 8 & $<1$ & 1024 & $>1024$ & 64 & 16 & 1204 & 32 \\
\hline 84 & 3.0 & 0.04 & 64 & 16 & 512 & 1024 & 8 & 256 & 128 & 128 & 1024 & $>1024$ & 8 & 512 \\
\hline
\end{tabular}

norfloxacin (NOR); ciprofloxacin (CIP); amikacin (AMK); ceftriaxone (CRO); gatifloxacin (GAT); cefepime (FEP)

Among the tested P. mirabilis strains, three multidrug resistant strains were found (no. 34, 70 and 84), against which none of the tested antibiotics was active.

The MBEC assay was used to examine the ability of the tested bacteria to form biofilm on polystyrene surface (Table III and IV). All studied uropathogens formed biofilm on polystyrene pegs - P. mirabilis with density from $0.04 \times 10^{6}$ to $9.91 \times 10^{6}$ cells per peg (the most intensive growth was observed for strains no. 70, $71,46)$ and in the case of the other uropathogens the average density was, for example, $6.78 \times 10^{6} \mathrm{CFU} / \mathrm{peg}$ for P. stuartii and $0.47 \times 10^{6} \mathrm{CFU} /$ peg for E. cloacae.

Six antibiotics were used to study biofilm sensitivity (norfloxacin, ciprofloxacin, amikacin, ceftriaxone, gatifloxacin, cefepime). The distribution of MICs and MBECs is presented in Table III. Significant differences between MICs and MBECs values of most antibiotics were detected. The MBECs were often more than 1000-fold higher than the MICs. The most effective drugs in planktonic cell eradication occurred to be amikacin and ceftriaxone (16 of 22 strains were susceptible). 12 strains were vulnerable to cefepime. Some activity of norfloxacin, ciprofloxacin and gatifloxacin was also observed. Similarly to the results obtained in the microdilution method, three strains were found to be resistant to all the tested antibiotics (P. mirabilis 34, 70 and 84 ). It was revealed that $100 \%$ of P. mirabilis sessile forms were resistant to norfloxacin, gatifloxacin, cefepime and ciprofloxacin. Amikacin and ceftriaxone inhibited only two P. mirabilis strains (5\%). The correlation between $P$. mirabilis sessile forms sensitivity to drugs and biofilm intensity was not observed.

E. coli, P.aeruginosa, K. pneumoniae, P. stuartii, E. cloacae and M. morganii were isolated from urinary catheters as P. mirabilis associated flora. The susceptibility of these rods to the tested drugs was determined and compared to P. mirabilis sensitivity. MICs and MBECs values of the studied uropathogens are presented in Table IV.

The planktonic cells of the uropathogens were mostly vulnerable to the tested drugs e.g. M. morganii strains were sensitive to all antibiotics. K. pneumoniae strains were resistant only to gatifloxacin; E. cloacae to ceftriaxone and ciprofloxacin (strain no.31). A different observation was made for $P$. aeruginosa strains, which in high concentrations were resistant to all the tested 
Table IV

Resistance of studied uropathogens to antibiotics.

\begin{tabular}{|c|c|c|c|c|c|c|c|c|c|c|c|c|c|c|}
\hline \multirow{2}{*}{ Strains } & \multirow{2}{*}{$\begin{array}{c}\text { Inoculum } \\
\times 10^{7} \\
\mathrm{CFU} / \mathrm{ml}\end{array}$} & \multirow{2}{*}{$\begin{array}{l}\text { Biofilm } \\
\times 10^{6} \\
\mathrm{CFU} / \mathrm{ml}\end{array}$} & \multicolumn{6}{|c|}{$\begin{array}{l}\text { Resistance of planktonic } \\
\text { population (MIC) }\end{array}$} & \multicolumn{6}{|c|}{ Biofilm resistance (MBEC) } \\
\hline & & & NOR & CIP & AMK & $\mathrm{CRO}$ & GAT & FEP & NOR & CIP & AMK & $\mathrm{CRO}$ & GAT & FEP \\
\hline $\begin{array}{l}\text { K.pneumoniae } \\
46\end{array}$ & 6.30 & 4.31 & $<1$ & $<1$ & 4 & $<1$ & 128 & $<1$ & 256 & 128 & 128 & 32 & 256 & 16 \\
\hline $\begin{array}{l}\text { K. pneumoniae } \\
56\end{array}$ & 8.80 & 0.50 & $<1$ & $<1$ & $<1$ & $<1$ & $<1$ & $<1$ & 512 & 32 & $<1$ & 32 & $<1$ & 4 \\
\hline P. aeruginosa 11 & 3.40 & 1.50 & 1024 & 1024 & $>1024$ & $>1024$ & 256 & $>1024$ & 1024 & 1024 & $>1024$ & $>1024$ & 1024 & $>1024$ \\
\hline P. aeruginosa 46 & 5.20 & 0.94 & 128 & 128 & 512 & 1024 & 512 & $>1024$ & 512 & 512 & 1024 & $>1024$ & 1024 & $>1024$ \\
\hline P. stuartii 9 & 0.80 & 2.78 & 256 & 128 & 8 & 4 & 512 & 8 & 1024 & 1024 & 32 & 256 & 1024 & $>1024$ \\
\hline P. stuartii 71 & 4.30 & 10.78 & 16 & 8 & 8 & 8 & 4 & $<1$ & 1024 & 1024 & 1024 & 128 & 512 & 512 \\
\hline M. morganii 67 & 12.90 & 0.70 & $<1$ & $<1$ & 4 & 4 & $<1$ & $<1$ & 64 & 32 & 32 & 32 & 16 & 16 \\
\hline M.morganii 42 & 12.30 & 0.60 & $<1$ & $<1$ & 4 & 2 & $<1$ & $<1$ & 64 & 8 & 8 & 8 & $<1$ & 32 \\
\hline E. cloacae 31 & 5.40 & 0.28 & $<1$ & $<1$ & 2 & 256 & $<1$ & 16 & 128 & 32 & 2 & 128 & 32 & 256 \\
\hline E. cloacae 36 & 8.40 & 0.66 & 4 & 4 & 4 & 128 & 2 & $<1$ & 64 & 4 & 4 & 64 & 2 & 128 \\
\hline E. coli 9 & 15.20 & 1.31 & 512 & 512 & 16 & 16 & 8 & 2 & $>1024$ & $>1024$ & 1024 & 512 & 16 & 128 \\
\hline E. coli 71 & 12.0 & 2.20 & $<1$ & $<1$ & 2 & 2 & $<1$ & 128 & 128 & 16 & 1024 & 64 & 16 & 256 \\
\hline
\end{tabular}

norfloxacin (NOR); ciprofloxacin (CIP); amikacin (AMK); ceftriaxone (CRO); gatifloxacin (GAT); cefepime (FEP)

antibiotics. The most effective drugs against the uropathogens planktonic forms were: amikacin and cefepime.

Biofilms of the studied uropathogens (similarly to those of Proteus strains) were more resistant to the tested antibiotics than planktonic cells (MBECs were 2-512 fold higher than the MIC values), however, the drugs concentrations required for biofilm eradication were lower. Biofilms of M. morganii, E. cloacae and $K$. pneumoniae strains were more vulnerable to some antibiotics (e.g. M. morganii 42 was sensitive to amikacin, ceftriaxone and gatifloxacin, K. pneumoniae 56 to amikacin, gatifloxacin and cefepime). P. aeruginosa, P. stuartii and E.coli biofilms were resistant to all the tested drugs. There is no connection between biofilm thickness on the pegs and its susceptibility to antimicrobials.

\section{Discussion}

Catheter associated UTIs is very common bacterial disease in humans, specially in long time catheterized patients. The cause of this recurrent infections is bacterial biofilm, which coats the catheter surface and constitutes a reservoir of organisms resistant to host defense and drugs. In our research biofilms from 88 catheters, obtained from long term catheterized persons were isolated. About $50 \%$ of the isolates belonged to three species of bacteria: Pseudomonas aeruginosa, Enterococcus faecalis and E. coli. Klebsiella pneumoniae and Proteus mirabilis were also commonly detected, with the frequency of $20.7 \%$ and $17.9 \%$, respectively.
MacLeod and Stickler (2007) examined 106 urinary catheters, collected from hospitalized patients, and isolated a very similar composition of bacterial species (P. aeruginosa, E. faecalis, E. coli, and P. mirabilis). P. mirabilis isolation frequency was higher $-30.2 \%$ and that of K. pneumoniae was less frequent (17.9\%). When urine specimens collected from long therm catheterized or hospitalized patients were analyzed, a different composition of bacterial species was observed: E. coli were the most commonly isolated bacteria (70\% of samples), P. aeruginosa - 1.7-4\% and Enterococcus spp. - 2-5\% of samples (Ghadiri et al., 2012, Niveditha et al., 2012). Proteus isolates were detected with lower frequency (6.7\%), which may suggest that Proteus forms crystalline biofilms and bacterial cells are not easily released into urine. Our studies showed that Proteus mirabilis formed multi-species biofilm - $98 \%$ of all tested catheters were coated with biofilm consisting of several microorganisms species (2-7 species), only from two catheters single-species biofilm was recovered. Patients, from whom urinary catheters were isolated had been catheterized for a long period of time (from a few months to a few years), time of catheter replacement was different (usually two weeks) - these factors contributed to a number of bacterial species detected in biofilm. By comparison, in Stickler (2008) studies one species of microorganisms was isolated from about $28 \%$ of catheters. The species composition of mixed biofilms was diversified, however, P. mirabilis associated flora most often included E. faecalis and P. aeruginosa. We also observed frequent co-colonization of $P$. stuartii and P. mirabilis. These results confirm the studies described 
by MacLeod and Stickler (2007), who also detected Proteus together with E. faecalis and P. stuartii. It is probable that these microorganisms interact in a positive way and form mixed biofilm, which facilitates their survival in the unfavorable urinary tract environment.

It is widely known that P. mirabilis urinary tract infections are difficult to eradicate because these bacilli produce urease, which catalyzes the hydrolysis of urea to ammonia and carbon dioxide. Urea disintegration results in environment alcalization, ammonia contributes to changes in local environment and it is believed to cause damage to host cells (Jacobsenet al., 2008, Oki et al., 2010). The consequence of this process is an increase in the urine $\mathrm{pH}$ and the production of struvite and carbonate apatite crystals. This process in catheterized patients leads to encrustation and blockage of the catheter lumen - a common problem of long term catheterized persons (Stickler and Feneley, 2011). Wilde et al. (2013) observed that $24 \%$ of studied patients reported a few events of catheter blockage in two months, which increased treatment costs, due to the necessity of additional therapy. Interestingly, bladder irrigating (which is not recommended practice and was done by $42 \%$ of patients) contributed to catheter blockage (Wilde et al., 2013).

The consequences of $P$. mirabilis infections are dangerous and it is necessary to devise new, efficient methods, which can help control biofilm related infections. Unfortunately, antibiotics applied in therapy are often ineffective. The National Working Group for Rational Therapy and Prevention of Infections in Poland recommended to treat UTIs in the first place with fluoroquinolones and trimethoprim - sulfamethoxazole. To treat complicated urinary tract infections fluoroquinolones, aminopenicilins with a $\beta$-lactamases inhibitor are administered. Aminopenicilins with a $\beta$-lactamases inhibitor and cephalosporins are the first drugs prescribed to pregnant women with UTIs (Kupilas, 2006). The studied $P$. mirabilis strains were resistant to cotrimoxazole, amoxicillin with clavulanic acid (more than $80 \%$ of strains). $41 \%$ of the studied P. mirabilis strains were also resistant to ciprofloxacin. Ciprofloxacin is characterized by broad activity and reaches a high concentration in blood and UT tissues (Mierzynska and Niemir, 2009). All fluoroquinolones are commonly used to treat UTIs. They could penetrate the biofilm matrix e.g. ciprofloxacin penetrates into the biofilm structure within 20 minutes and obtains the MIC (Anderl et al., 2000). Furthermore, these antibiotics are active against slow growth cells so they are recommended to treat biofilm related infections (Passerini de Rossi et al., 2009).

Cephalosporins, very often recommended to treat UTIs, have great bactericidal activity with the exception of strains producing ESBL (Extended-Spectrum Beta-
Lactamases). $14.4 \%$ of P. mirabilis strains in Poland produce ESBL (Kwiecinska-Piróg et al., 2010). It has been proved that Proteus strains, synthesizing these enzymes and forming strong biofilm, are particularly dangerous and infections caused by them are persistent and difficult to treat (Nucleo et al., 2010). Most studied strains (planktonic populations) were vulnerable to these drugs, but their biofilms were resistant. Explanation for biofilm resistance could be the presence of $\beta$-lactamases, which are accumulated in the biofilm matrix - $\beta$-lactams antibiotics could be hydrolyzed before their penetration into biofilm (Hoiby et al., 2010). Spoering and Lewis (2001) suggest that biofilm resistance could result from the fact that microorganisms in biofilm have slow metabolism, similarly to bacteria in the stationary growth phase, while $\beta$-lactams are antibiotics which bind to fast growing cells. It is possible that cephalosporins (e.g. cefuroxime) activity relies only on delaying biofilm formation (Koseoglu et al., 2006, Spoering and Lewis 2001). Cefepime is characterized by stability against ESBL and AmpC enzymes and it is recommended to treat UTIs caused by ESBL - producing organisms (da Silva Nogueira et al., 2011). Bantar et al. (2004) suggest that substituting ceftriaxone with cefepime in UTIs caused by $P$. mirabilis results in a decrease in bacterial resistance to cephalosporins.

Aminoglycosides are recommended as an alternative to fluoroquinolones and cephalosporins to treat especially complicated UTIs (Kupilas, 2006). However, amikacin is not often used to treat UTIs because of dangerous side effects, despite the fact that its concentration in kidneys is high. The examined P. mirabilis planktonic cultures occurred to be sensitive to amikacin. The rare administration of this drug (usually in hospitals) in UTIs could be the reason for its high efficacy in P. mirabilis eradication. P. mirabilis biofilms were more resistant to this antibiotic contrary to biofilms of the other studied uropathogens (M. morganii, E. cloacae, K. pneumoniae). The extracellular matrix binds the drugs and inhibits penetration to deeper biofilm layers, which could be the reason why biofilms are resistant to aminoglycosides (Spoering and Lewis 2001). It is also probable that the oxygen limitation and the metabolic rate play an important role in this process (Hoiby et al., 2010).

Because of a higher rate of bacterial resistance to popular drugs, many urologists increasingly suggest a change of bad antibiotic-prescription behaviour. Trimethoprim/sulfomethoxazol and fluoroquinolones should not be recommended for therapy in the case of complicated UTIs (Mazzulli, 2012). Fosfomycin/ trometamol, nitrofurantoin, pivmecillinam should be current drugs of first choice (Wagenlehner and Naber, 2012). Pallett and Hand, 2010 also suggest alternative treatment e.g. combination of cefepime or cefpirome with clavulanate or fosfomycin. 
In conclusion, $P$. mirabilis is very often isolated from urinary catheters, where it forms encrusted, multispecies biofilm, resistant to the majority of drugs. Biofilm related infections are persistant and difficult to treat. It is important to administer antibiotics with care (e.g. appropriate drugs in adequate doses), because the consequence of badly conducted treatment could be the extension of bacterial resistance to antibiotics.

\section{Acknowledgments}

We would like to thank Bristol-Myers Squibb for providing cefepime and gatifloxacin samples and allowing us to use them in our study.

\section{Literature}

Anderl J., M. Franklin and P. Stewart. 2000. Role of antibiotic penetration limitation in Klebsiella pneumoniae biofilm resistance to ampicilin and ciprofloxacin. Antimicrob. Agents. Chemother. 44: 1818-1824.

Bantar C., E. Vesco, C. Heft, F. Salamone, M. Krayeski, H. Gomez, M.A. Coassolo, A. Fiorillo, D. Franco, C. Arango and others. 2004. Replacement of broad spectrum cephalosporins by piperacillintazobactam: impact on sustained high rates of bacterial resistance. Antimicrob. Agents. Chemother. 48: 392-395.

Ceri H., M. Olson, C. Stremick, R. Read, D. Morck and A. Buret. 1999. The Calgary Biofilm Device: new technology for rapid determination of antibiotic susceptibilities of bacterial biofilms. J. Clin. Microbiol. 37: 1771-1776.

Chen L. and Y. Wen. 2011. The role of bacterial biofilm in persistent infections and control strategies. Int. J. Oral. Sci. 3: 66-73.

da Silva Nogueira K., A. Vale Daur, I. Taborda de Messias, A.C. Gales and L.M. Dalla Costa. 2011. Cefepime versus extended spectrum $\beta$-lactamase-producing Enterobacteriaceae. Braz. J. Infect. Dis. 15: 167-169.

Ghadiri H., H. Vaez, S. Khosravi and E. Soleymani. 2012. The antibiotic resistance profiles of bacterial strains isolated from patients with hospital-acquired bloodstream and urinary tract infections. Crit. Care. Res. Prac. doi:10.1155/2012/890797.

Hoiby N., T. Bjarnsholt, M. Givskov, S. Molin and O. Ciofu. 2010. Antibiotic resistance of bacterial biofilms. Int. J. Antimicrob. Ag. 35: 322-332.

Jacobsen S.M., D.J. Stickler, H.L.T. Mobley and M.E. Shirtliff. 2008. Complicated catheter associated UTI due to Escherichia coli and Proteus mirabilis. Clin. Microbiol. Rev. 21:26-57.

Koseoglu H., G. Aslan, N. Esen, B. Sen and H. Coban. 2006. Ultrastructural stages of biofilm development of Escherichia coli on urethal catheters and effects of antibiotics on biofilm formation. Urology 68: 942-946.

Kupilas A. 2006. Urinary tract infections. Przegl. Urol. (in Polish). 4: 50-54.

Kwiecińska-Piróg J., T. Bogiel and E. Gospodarek. 2010. The incidence of extended spectrum beta-lactamases in Proteus mira- bilis strains isolated in 2007-2009. Przegl. Epidemiol. (in Polish). 4: 395-398.

Lazar V. and MC. Chifiriuc. 2010. Medical significance and new therapeutical strategies for biofilm associated infections. Roum. Arch. Microbiol. Immunol. 69 (3): 125-38.

MacLeod S. and D.J. Stickler. 2007. Species interactions in mixedcommunity crystalline biofilms on urinary catheters. J. Med. Microbiol. 56: 1549-1557.

Mazzulli T. 2012. Diagnosis and management of simple and complicated urinary tract infections (UTIs). Can. J. Urol. 19: 42-48.

Mierzynska E. and Z.I. Niemir. 2009. Antibiotics in nephrology (Part II) - principles of treatment of the most frequent diseases and their complications in hospitalized patients. Contemp. Pharm. 2: $102-109$.

Muzzi-Bjornson L. and L. Macera. 2011. Preventing infection in elders with long-term indwelling urinary catheters. J. Am. Acad. Nurse. Prac. 23: 127-134.

Niveditha S., S. Pramodhini, S. Umadevi, K. Shailesh and S. Selvaraj. 2012. The isolation and biofilm formation of uropathogens in the patients with catheter associated urinary tract infections (UTIs). J. Clin. Diagn. Res, 6: 1478-1482.

Nucleo E., G. Fugazza, R. Migliavacca, M. Spalla, M. Comelli, L. Pagani and M. Debiaggi. 2010. Differences in biofilm formation and aggregative adherence between $\beta$-lactam susceptible and $\beta$-lactamases producing P. mirabilis clinical isolates. New. Microbiol. 33: 37-45.

Oki K., K. Washio, D. Matsui, S. Kato, Y. Hirata and M. Morikawa. 2010. The role of urease activity on biofilm formation by Staphylococcus sp. T-02 isolated from the toilet bowl. Biosci. Biotechnol. Biochem. 74: 583-589.

Pallett A. and K. Hand. 2010. Complicated urinary tract infections: practical solutions for the treatment of multiresistant Gram-negative bacteria. J. Antimicrob. Chemother. 65 Suppl. 3: 25-33.

Passerini de Rossi B., C. García, M. Calenda, C. Vay and M. Franco. 2009. Activity of levofloxacin and ciprofloxacin on biofilms and planktonic cells of Stenotrophomonas maltophilia isolates from patients with device-associated infections. Int. J. Antimicrob. Ag. 34: 260-264.

Spoering A. and K. Lewis. 2001. Biofilms and planktonic cells of Pseudomonas aeruginosa have similar resistance to killing by antimicrobials. J. Bacteriol. 183: 6746-6751.

Stickler D.J. 2008. Bacterial biofilms in patients with indwelling urinary catheters. Nat. Clin. Pract. Urol. 5: 598-608.

Stickler D.J. and R.C.L. Feneley. 2011. The encrustation and blockage of long-term indwelling bladder catheters: a way forward in prevention and control. Spinal. Cord. 48: 784-790.

Wagenlehner F. and K. Naber. 2012. Prescribing behaviour in urinary tract infection. Dtsch. Arztebl. Int. 109: 876-877.

Wilde M., M. McDonald, J. Brasch, J. McMahon, E. Fairbanks, S. Shah, W. Tang and E. Scheid. 2013. Long-term urinary catheter users self-care practices and problems. J. Clin. Nurs. 22: 359-367.

Yang L., Y. Liu, H. Wu, N. Hoiby, S. Molin and Z. Song. 2011. Current understanding of multi-species biofilms. Int. J. Oral. Sci. 3: 74-81. 